\title{
Governance and Quality Award at the ENMS of León
}

\author{
Mónica Mondelo Villaseñor, Juana Beatriz Ortiz Ciénega \\ ENMSL, University of Guanajuato, México
}

\begin{abstract}
The "Escuela de Nivel Medio Superior de León" (ENMSL), is a public high school located in León, one of the largest cities of Guanajuato State and belongs to the University of Guanajuato, in México; in recent years we have taken part in the "Sistemas de Gestión de la Calidad" (SGC) program, a Quality Assurance and Management System based on the ISO 9001:2008/NMX-CC-9001-IMNC-2000, which has allowed us to obtain the certificates No. MX08/19389 for ISO 9001:2008 and No. GTO-ISO001 for GTO-2000 granted by the Société Générale de Surveillance (SGS) and the Guanajuato Institute for Quality and Competitiveness (IGC) respectively. Besides the ISO 9001:2008 certification, we participated in the best quality practices of the León Metropolitan for Quality Award (PMLC). Having this previous achievements, it was decided to carry out this research with the purpose of carrying out the present comparison study between the two aforementioned models, supported by the methodology provided by the model of the international standard ISO 10014: 2006 / NMX-CC10014-IMNC-2008 Quality Management Guidelines for obtaining financial and economic benefits and the PMLC total quality model-. The ENMSL has an acceptable level of compliance with maturity in reference to the eight management principles, as well as monitoring and control activities in the academic and administrative processes to reach world class levels.
\end{abstract}

\section{Introduction}

The development of institutions sustainable success is managed by participation and intervention of total quality systems, wherewith, it is tried to demonstrate the fulfillment of the quality management principles using a maturity level measuring grades.

Total quality models, global, national and local, help and encourage organizations to make a selfmeasurement performance exercise related to standardized criteria. That's why the ENMSL staff needs to participate in the León Metropolitan Quality Award (PMLC).

The PMLC is an award of total quality granted by institutions that represent the society as a whole, which will allow the ENMSL to have a feedback and in its case an acknowledgment of compliance of the model and requirements for the prize given. The ENMSL is currently facing a several factors that can put to an end its high ranked position of quality and social prestige as an institution.

These factors are, among others are, the large number of recent high schools that compete and their efforts to develop and placing themselves in highlevel educational institutions; the resistance to be democratic and to attend regulations as a way to organize ourselves in a state of law that allows us to work and coexist in fair competition and the presence of psychosocial risks for teenagers.

Based on the opinion polls conducted among the high school community, the priorities that arise, and what the participation in the PMLC will be focused are:

To practice a leadership that undertakes actions for the continuous improvement in academic, administrative, cultural and sports programs that can lead to the integral formation of the student.

The administrative and teaching staff teamwork to develop of attitudes that imply making emotional, intellectual and labor commitments to contribute to the achievement of institutional purposes.

The consolidation of the ENMSL as the best alternative for high school education.

The challenge today is preserve the status earned over more that twelve decades, which depends on the capability as an institution to train free and responsible men and women with a competitive academic level, achieved all this with the support of the processes established by the quality standards that are already implemented and those that will be sought will be established in the near future.

The high school educational offer of the University of Guanajuato consist of eleven campuses, all together they form the "Colegio de Nivel Medio Superior" (High School College) of our University. A high percentage of its students has been distinguished for the good academic level, certified by external agencies and by its constant improvement efforts, making the necessary steps to enter the National Baccalaureate System (SNB).

\section{Theoretical frameworks}

\subsection{Quality management and total quality models}

The graphic representation shows the generic model of the global process for obtaining financial and economic benefits (PMLC). 


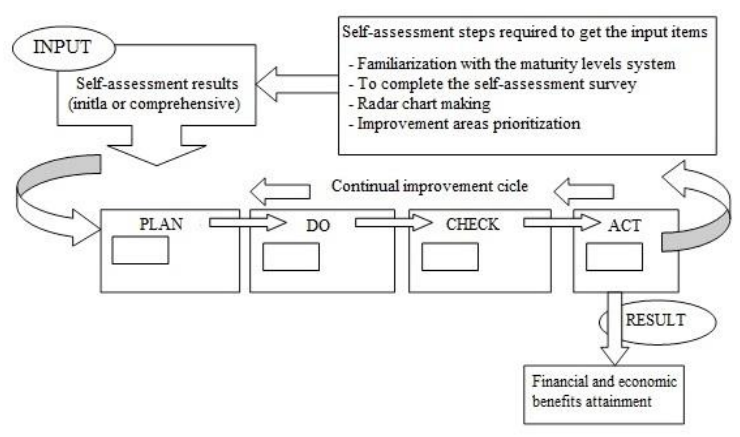

Figure 1. Global representation process to obtain financial and economic benefits

(Source: ISO 10014: 2006 / NMX-CC-10014-IMNC-2008 Quality Management - Guidelines to obtain financial and economic benefits)

Indicates the flow of monitoring and control to be followed in four moments, in which is established as a process-based approach, the previous steps to follow where the information is obtained that will serve as input for the self-assessment (initial and/or comprehensive) result and as a transformation process the cycle of continuous improvement established in the methodology PDCA (ISO, 2008) in each of the eight management principles, as well as the methods and tools to be used in them and finally as a result or the economic and financial benefits expected for the institution in the process.

In reference to the Participation Guide of the León Metropolitan Quality Award.

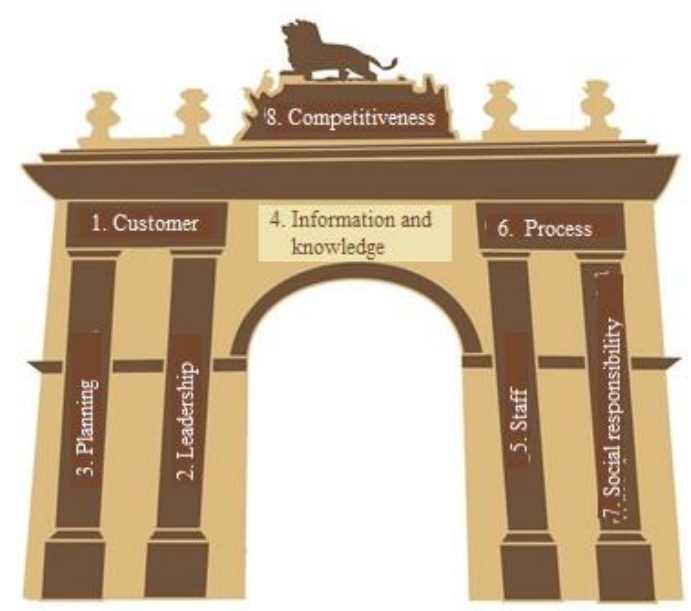

Figure 2. Criteria of the PMLC (Source: PMLC Participation Guide)

\section{Conceptual theoretical framework}

This context is established in the eight principles of quality management system (ISO, 2001) and the eight criteria of the PMLC that are part of both the quality system and total quality basis.
From this eight models principles, four of them are aligned such as: Customer Focus, Leadership, Staff Involvement and Process-based Approach.

Customer focus: Organizations depend on their customers and therefore should understand the current and future needs of them, meet customer/client requirements and strive to exceed the customer expectations

Leadership: Leaders establish the organization purpose and. They must create and maintain an internal environment, in which staff can become fully involved to achieve the organization goals.

Staff involvement: Staff, at all levels, are the essence of an organization, and its Total commitment enables their skills to be used for the benefit of the organization.

Process-based approach: A desired result is achieved more efficiently when the activities and related resources are managed as a process.

The remaining eight that are not aligned are: Systematic approach to management, Continual improvement, Factual approach to decision making, Mutually beneficial supplier relations, Planning, Information and knowledge, Social responsibility and Competitiveness of the organization.

Systematic approach to management: Identify, understand and manage interrelated processes as a system, it contributes to the effectiveness and efficiency of an organization in achieving its objectives.

Continual improvement: Continual improvement of the overall performance of the organization should be an active and permanent objective.

Factual approach to decision making: Effective decisions are based on analysis of data and information.

Mutually beneficial supplier relations: An organization and its suppliers are interdependent, and a mutually beneficial relationship increases the ability of both to create value.

Planning: It is the way in which planning guides your organization towards the improvement of its competitiveness, including the way in which its objectives and strategies are defined and how they are deployed in the organization depending on the possible future scenarios of the city.

Information and knowledge: Includes the mechanism used by the organization to obtain, structure, communicate and analyze information and knowledge for the administration of processes and support strategies, as well as the development of the organization.

Social responsibility: It includes the way in which the organization assumes its social responsibility to contribute to the sustainable development of its environment and the promotion of quality culture in the community. 
Competitiveness of the organization: Evaluates the performance of the organization in the creation of value towards its different interest groups, as well as the causal relationship between the key indicators of the business and the processes and system of the organization.

\section{Goals}

\subsection{General}

The fundamental purpose of the research is to compare the results of the implementation of Management Models ISO 10014: 2006 / NMX-CC10 014-IMNC-2008 of Quality Management Guidelines for obtaining financial benefits and the Total Quality Model of the León Metropolitan Quality Award in ENMSL.

\subsection{Individual}

1. Compare the elements and criteria of the ISO 10014: 2008 Quality Management Models and the León Metropolitan Quality Award.

2. To link the methodologies, methods, guide tools and guidelines of ISO 10014: 2008 and the León Metropolitan Award for Quality.

\section{Problem Approach}

In this case-study research comparative between the two models of quality management, it is intended to perform the analysis of the quality fundamentals, such as the eight principles of quality management and criteria of the PMLC, respectively in its application in the ENMSL.

Are the principles of the quality management system and the criteria of the total quality model of the PMLC identical in their application in the ENMSL?

\section{Method}

The comparison method to meet the aforementioned objectives is established below:

The first model consisted of four phases or stages, such as the steps of self-evaluation to achieve the input element, input, process (methodology PDCA methods and tools) and results (output) economic and financial benefits. The activities or tasks were: description of the maturity levels, questionnaires for the initial and exhaustive self-evaluation, radar chart, improvement-prioritization opportunities, initial and comprehensive self-assessment results, continual improvement cycle and conclusions.

The second model consisted of a three-stage evaluation process called short reporting, system reporting and field visit.
Short report: Description of the basic profile of the organization: organization chart, number of employees, nature of the organization, provided services, key success factors, strategic relationships with other organizations, suppliers, main facilities and technologies, legal framework in aspects such as environmental, social security, that have an impact in the organization, competitiveness results of the organization and description of quality practices according to the eight criteria of the PMLC model.

System reports: Systemic description of the quality processes of organizations that qualify for this phase. It is carried out through the evaluation of an extensive and detailed document presented by the organizations, based on the eight criteria and subcriteria of the PMLC model.

Field visit: Corroborate and expand, through evidence, the information presented in the previous documented stages. Answer questions that may have arisen during the previous stages. To define the maturity and placement levels of the organization's quality system.

The information resources used in the research were strictly the international standard ISO 10014: 2006 / NMX-CC-10014-IMNC-2008 of Quality Management - Guidelines to obtain financial and economic benefits and the PMLC participation Guide.

\section{Instruments}

\subsection{Questionnaire application for the initial self-assessment}

To fulfil the surveys, both initial and comprehensive, the academic and administrative staff serve as actors in the questionnaires application, previously received a training course for knowledge and understanding of the five levels and description of the established maturity in the annex of Table 1, as well as the questions contained in the initial questionnaire, established in the guideline standard used as methodology in the investigation.

Table 1. Maturity level matrix - absolute relationship vs. percentage, initial survey

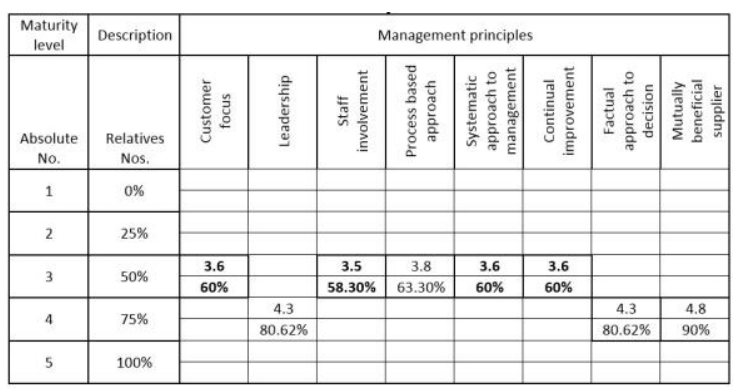

(Source: Own elaboration, based on data from the initial surveys, $2014-2015$ 
At each level of maturity, the content is accurately described for your understanding, as well as the relationship between the level of maturity in absolute number, from number one to five, as well as your correspondence in relative or percentage number of the percentage number, from zero to one hundred percent.

The questionnaire for the initial self-assessment is composed of the eight quality management principles as titles and three questions referenced in relation to them, in such a way that a total of twentyfour questions were answered.

Once the questionnaires were answered, they were processed in an electronic database, with the purpose of obtaining the figure graph or radar diagram and showing in a visual way the behavior of the different perceptions in relation to the level of maturity that it occupies in the range of zero to five of the management principles, as shown in Figure 3 Radar chart - management principles, initial surveys; which shows that the lowest score with 3.5 belongs to the management principle "staff involvement", although it has a behavior of one point above the average (2.5), is one point and a half short to obtain the maximum level of maturity (five).

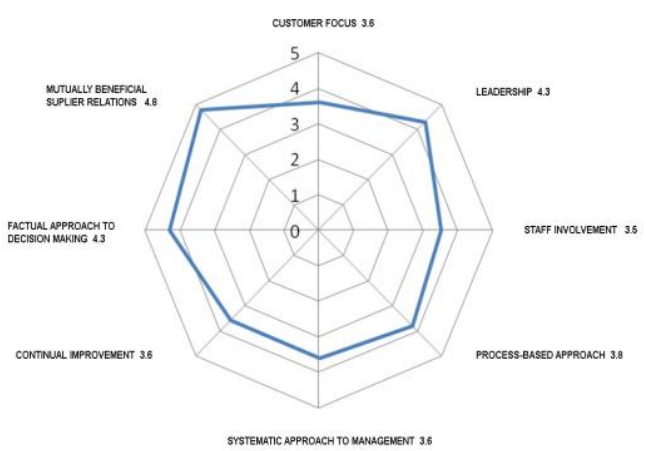

Figure 3. Radar chart, management principles initial survey

(Source: Own elaboration, based on initial survey, 2014 -2015)

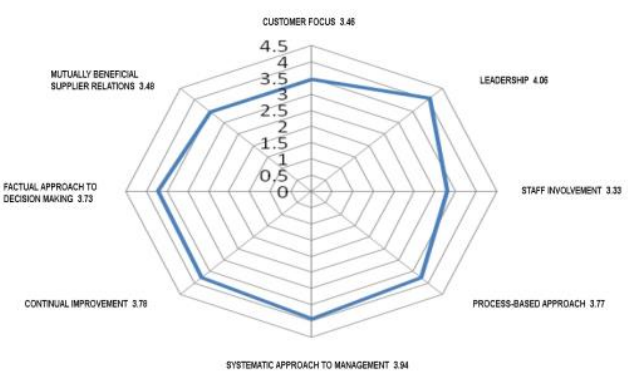

Figure 4. Radar chart, management principles, comprehensive survey

(Source: Own elaboration, based on comprehensive survey, 20142015)
We did use the Vilfredo Pareto 80/20 paradigm, in which we consider that resolving 20 percent of the causes of the problems in the organizations can help solve the remaining 80 percent, we would have to intervene in four of the eight principles, such as staff involvement (level 3.5), customer focus, systematic approach to management and continual improvement (level 3.6 respectively).

The best evaluated were the management principles, process-based approach (3.8), leadership and factual approach to decision making (4.5 respectively) and mutually beneficial supplier relations (4.8 the highest). It would seem that in absolute terms the level presented of the selfassessments reflect a satisfactory degree of the management principles in the ENMSL; analyzing the Table 2, Matrix of maturity level - absolute relationship vs. percentage, - initial survey; from the point of view of relative or percentage numbers, the five management principles that occupy the level of maturity (3) represent between 58 to 63 percent of the level of one hundred percent and only the three remaining management principles that occupy the level of maturity (4) represent between 80 to 90 percent of the level of one hundred percent. Because of the above, we have barely a passing point, so as not to be faulted.

Table 2. Maturity level Matrix, absolute relationship vs. percentage, comprehensive survey

\begin{tabular}{|c|c|c|c|c|c|c|c|c|c|}
\hline $\begin{array}{c}\text { Maturity } \\
\text { level }\end{array}$ & Description & \multicolumn{8}{|c|}{ Management principles } \\
\hline $\begin{array}{l}\text { Absolute } \\
\text { No. }\end{array}$ & $\begin{array}{l}\text { Relatives } \\
\text { Nos. }\end{array}$ & 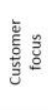 & 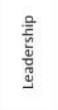 & 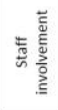 & 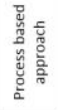 & 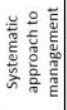 & 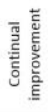 & 要 & 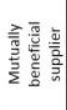 \\
\hline 1 & $0 \%$ & & & & & & & & \\
\hline & & & & & & & & & \\
\hline 2 & $20 \%$ & & & & & & & & \\
\hline 3 & $50 \%$ & 3.46 & & 3.3 & 3.77 & 3.94 & 3.78 & 3.73 & 3.48 \\
\hline & & $58 \%$ & & $55.00 \%$ & $62.83 \%$ & $66 \%$ & $63 \%$ & $62.16 \%$ & $58 \%$ \\
\hline 4 & $75 \%$ & & 4.06 & & & & & & \\
\hline 4 & & & $76.13 \%$ & & & & & & \\
\hline 5 & $100 \%$ & & & & & & & & \\
\hline & & & & & & & & & \\
\hline
\end{tabular}

(Source: Own elaboration, based on data from the comprehensive surveys, 2014-2015)

\subsection{Questionnaire application for exhaustive self-assessment}

The radar charts provide a graphic vision of the level or state of maturity of the ENMSL in reference to the eight management principles, likewise, it illustrates the continuous progress of the institution.

Maturity level matrix - absolute relationship vs. percentage - of the surveys, both initial and comprehensive, show a more precise analysis in relation to the performance achieved in the use of the 
eight principles of quality management in the ENMSL.

In a second time and having as a premise the training in reference to the levels of maturity and questions of the comprehensive self-assessment questionnaires, the comprehensive survey was applied, which contains 76 questions related to each of the eight management principles.

Again, the management principles "staff involvement" and "customer focus" got the lowest score (3.3 and 3.4 respectively), as well as showed a downward adjustment with respect to the initial selfassessment.

The only management principle that remained at the level of maturity (4) was "leadership", while the "Factual approach to decision making" and "Mutually beneficial supplier relations" principles fell from the level of maturity (4) to level (3) respectively.

Overall, in both initial and comprehensive surveys, the perception of academic and administrative staff in reference to the use of the eight principles of quality management in the ENMSL, are located at the maturity level (3) and the only one that shows a tendency to remain at the level (4) is the management principle "Leadership".

The upper management of the institution, should implement and apply the "Factual approach to decision making" quality management principle, in reference to the eight management principles, in order to carry out activities that allow to grow at the levels of maturity from (3) to (5).

Some results that the ENMSL can obtain from the application of the management principles are, among others, increased competitiveness, improved customer retention and loyalty, optimization of available resources, increased employee responsibility, improvement of intellectual assets, effectiveness and efficiency of processes optimization, sustainability of the institution.

\section{First Stage of the PMLC}

\subsection{Customer satisfaction}

\subsubsection{Knowing the Customer and market.}

Strong areas: There is a procedure for measuring customer satisfaction, which allows the Institution to monitor the satisfaction of its customers with quantitative indicators.

Improvement areas: Anticipating the needs and expectations of different groups of customers/end users, current/potential, medium/long term, will allow the organization to position itself in its local and regional market.

It is necessary to determine the service critical factors of the different customers to identify the importance they have for their choice making, this is important for a possible service expansion.
The satisfaction survey provides very information to meet the needs and expectations of customers (students) but does not identify potential end users in the medium and long term.

Satisfaction is measured through the satisfaction survey, applied to the client every six months; it is not described if loyalty can or cannot also measured through this mechanism.

\subsubsection{Integral Relationship with Clients.} Improvement areas: Lack of data that describe the way in relations with their customers are managed, so that could be strengthened and increase loyalty, and by that potential customers choose our high school over the competence.

Information from the satisfaction survey, complaints and suggestions is collected, however the mechanism of how it displays the detected requirements is not established.

A mechanism to get the complaints and suggestions exist but does not clarify how they are solved quickly and effectively to ensure the satisfaction and increase their confidence in the services received.

The needs of the customer are known through the satisfaction survey, but it is necessary to specify if that means to provide help, advice, information or the customer is expressing their concerns when receiving the services of the ENMSL.

\subsection{Leadership}

Strong areas: The high school shows a process map where its processes and interactions are described.

Improvement areas: It has defined mission, vision, policy and quality objectives, having implemented a quality management system, overall performance of its system must and will be evaluated at least once a year.

It is necessary to establish a mechanism to promote and evaluate the organizational culture that encourage to act in accordance with the ENMSL principles. It is still necessary to define the groups of interest that the ENMSL has.

The mechanisms to communicate the most relevant information to achieve the staff involvement and benefit from their suggestions are not observed, this could help to develop a profile of leadership skills throughout the ENMSL.

\subsection{Planning}

\subsubsection{Strategic Planning}

Strong areas: The strategic planning is held in the 2010-2020 development project, where goals are set, which allows competitiveness improvement of the ENMSL. 
The objectives and priority goals, and their accomplishment time are established.

Improvement areas: There is no information about how strategic planning is made or what type of data is required to map out the evolution of the high school.

\subsubsection{Operational Planning}

Strong areas: The annual operational projects are aligned with the annual planning which creates certainty towards reaching the objectives.

Through the Academic and Administrative Improvement Program (PROSAA) accepted by the financial management and after receiving the budget cap information, the work on the annual operational projects begins; but the how to determine each operative project is not described.

Improvement areas: The objective is to design and implement an evaluation and monitoring system for annual operating plans.

\subsection{Information and Knowledge}

\subsubsection{Information}

Improvement areas: The informational content on the official website of the University of Guanajuato has no description of how was selected or obtained, meaning that can't be used to monitor the operations of the ENMSL and evaluate its performance. Nor is the reliability, timeliness and consistency of the supported data.

Although the information can be found on the website, it does not identify the ways in which this information can be accessed by internal and external users for choice making purposes.

\subsubsection{Organizational Knowledge}

Improvement areas: Besides the process knowledge for the services development of the ENMSL, a design mechanism that allows to identify the knowledge required by the staff to improve and increase the organizational matters must be designed. In addition, create an environment where innovation and creativity prevail is needed also.

To design and implement a mechanism to manage and allow access to this knowledge that was generated by innovation so that they can be used by other areas or even share them as best practices, this would create a competitive environment and improve the technological development in the ENMSL.

\subsection{Staff}

\subsubsection{Work Systems}

Strong areas: The ENMSL organization chart shows that its work systems are developed by a coordination board, defining the responsibilities and authority of itself; the design is focused on achieving a high-performance process and the achievement of the of the ENMSL goals.

Through the human resources process they identify, select and incorporate new staff in relation to labor competencies; a Training Need Detection program (DNC) is always active to keep developing the competitive feature of the ENMSL.

\subsubsection{Human Development}

Strong areas: The job profiles of the activities carried out according to the organization chart have been identified and defined properly.

The detection of training needs is used to reduce the gap between the current knowledge, skills and abilities, and the ones required in the short and long term. Set up conditions and practices are developed for continuous learning improvement.

Improvement areas: A mechanisms to create conditions and practices to reinforce continuous learning for the work systems improvement to increase the competitiveness of the ENMSL.

\subsubsection{Life Quality}

Strong areas: For the basified staff, the institution offers incentives for teachers who develop their skills, in addition, medical network and social security services are provided by Social Security Institute of the State of Guanajuato (ISSEG) for both administrative a teaching staff. These are few of the benefits to have a better life quality, however it is still necessary to create a stronger life quality system and that fully guaranties a family well-being.

Improvement areas: The systems or mechanisms required to define, evaluate and measure the critical satisfaction factors of ENMSL staff needs to be identified/developed, and what actions will be implemented to respond to the identified needs.

By reactivating the Occupational Safety and Health committee, they can design the mechanisms to promote safety, health, hygiene and ergonomics to all of the ENMSL activities.

\section{Processes}

\subsection{Design of Products, Services and Processes}

Strong areas: The Institution shows a process map where its processes and interactions are properly described.

Improvement areas: The needs and expectations of the customers are clear thanks to the satisfaction survey, however, mechanisms to turn them into service specifications and useful features are not identified in order to specify a value proposal for the organization.

No mechanism is identified for the design, redesign or improvement of processes that assures a 
consistent and fault-free performance to respond to the strategic needs of ENMSL.

\subsection{Process Administration}

Strong areas: The Institution have a certificated ISO 9000 and a GTO-ISO-001 system, which allows the assurance of activities, functions and processes in a standardized manner to assure a proper processes administration.

\section{Social responsibility}

\subsection{Ecosystems}

Improvement areas: Through the environmental training program they can give solution to this principle, however it is necessary to describe how they will develop each of the project such as:

Environmental impact of their processes, resources optimization, ecosystems protection and recovery promotion, provide environmental education.

\subsection{Community Development}

Improvement areas: The activities that are carried out for the development of the community are part of the daily work, in addition to what is done to generate permanent social welfare of their communities of influence, promote and participate with the community in processes of continuous improvement and share practices in favor of greater competitiveness in the industrial sector, business and the economy in general.

\section{Results}

Improvement areas: It is necessary to place indicators referring to customers, staff, society and shareholders with referential comparisons and the causal relationship of the conditions that have been presented in a favorable or unfavorable trend.

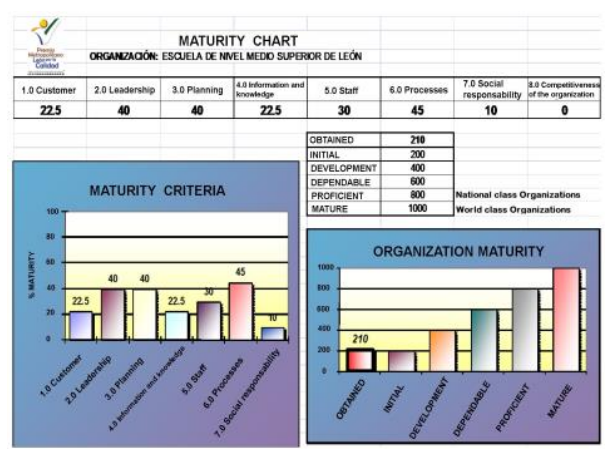

Figure 5. Maturity chart $1^{\text {st }}$ stage Source: PMLC 1st stage evaluation report.

\section{Second Stage of the PMLC}

\subsection{Customer Satisfaction}

11.1.1. Knowledge of Customers and Markets. Improvement areas: Only the student is identified as a customer and its population segment is only of 2000 students, not knowing the percentage that represents the entire market, as well as the market segment that the competition has; knowing this, would allow knowing differentiating aspects of the competition and that will serve as feedback or benchmark comparison for the ENMSL.

Identifying the needs and expectations of the customers would give them the opportunity to define the critical factors of the service of the different customers and point out the importance they have for their preference, serving this for a possible expansion.

The satisfaction survey provides information to know the needs and expectations of customers (students), but the necessary mechanisms to know and anticipate their needs have not been identified; which would allow a greater number of customers to prefer their services and the opportunity of an expansion with some linking centers in the city.

Customer satisfaction is measured through the satisfaction survey, which is applied semiannually; however, it is necessary to know the level of loyalty of the students, which would serve to identify the critical factors that the customer has.

11.1.2. Integral Relationship with Customers. Improvement areas: The mechanisms to strengthen and increase the loyalty of customers towards ENMSL, are beginning to be implemented. This will allow to obtain valuable information of received and expected service, as well as to define the critical factors of these.

Although information on complaints and suggestions is obtained through the satisfaction survey, there is no mechanism to solve the problem effectively and quickly, that makes it difficult for customers to easily recover their trust.

The client's needs are known through the satisfaction survey; however it is not identified how the customers are supported when they asks for help, advice or expresses they concerns regarding the services received from the ENMSL. The implementation of these mechanisms will allow to create loyalty in their customers.

\section{Leadership}

\subsection{Leadership}

Strong areas: It has defined the mission, vision, policy and quality objectives, as well as the 
implementation of a quality management system evaluated through the management, which allows to make the appropriate decisions.

Improvement areas: The high school shows a map of processes where the interactions between them are described, showing a systemic approach of the ENMSL; however, it is not robust enough as established by PMLC.

It is necessary to implement a mechanism that promotes and evaluates the organizational culture of the ENMSL that allows knowing the gap that exists between the actions of your organization and the principles established by the ENMSL.

It is important to identify the groups of interest for the ENMSL and how it interacts with them; since they are a very important part in the decisions making process to define the direction of the organization, aiming to be more competitive in the market.

The internal communication devices used to share information of greater relevance are not observed, which would help to achieve a greater involvement of the staff and to take advantage of their suggestions, likewise, to develop a leadership profile throughout the organization, allowing to excel in the educational sector.

\section{Planning}

\subsection{Strategic Planning}

Improvement areas: There are contemplated goals within the strategic planning in the 2010-2020 development project, but it is not identified how this planning is being developed, nor the necessary information to carry it out and thus project the evolution of the ENMSL.

The objectives and goals are established, however their measurement and relation with the operational objectives do not; which would allow the organization a better way to prioritize the projects and focus the necessary resources to them.

\subsection{Operational Planning}

Strong areas: Through the PROSAA, accepted by the financial management and receiving the budget cap information, work on the annual operational projects begins; but the how it determines each operative project is not described.

Improvement areas: The strategic objectives and the operative ones are not clear in their alignment; this can generate confusion and deviation of goals. The annual operational projects are aligned with the annual planning, however their alignment with the strategic planning is not identified.

The objective is to design and implement a system for monitoring and evaluating annual operating plans, this will prioritize plans that make the services offered more efficient and, in the long term, develop much more effective plans.

\section{Information and Knowledge}

\subsection{Information}

Improvement areas: The information generated is supported on the website of the University of Guanajuato, however it is not described how it is selected, the operations of the ENMSL are followed up and their performance is evaluated. Neither is the way in which the reliable, timely and consistent nature of the backed data is identified; which would help the ENMSL to make decisions more quickly for a continuous improvement of the institution.

The information found on the website is protected through a username and password; however, it does not describe mechanisms for external users.

\subsection{Organizational knowledge}

Improvement areas: In addition to the process's knowledge for the services development in the ENMSL, a mechanism could be designed to allow them to identify the knowledge required by the staff to improve and increase the organizational matters. In addition, to create an environment where innovation and creativity prevail. Also design and implement mechanisms to manage and allow access to this knowledge generated from innovation so that they can be used by other areas or even share them as best practices, this would create a competitive environment and improvement in technological development in the ENMSL.

\section{Staff}

\subsection{Work Systems}

Strong areas: Through the organization chart of the ENMSL, it is observed that the work systems are developed by coordination defining the responsibilities and authority of this; the design is focused on achieving a high performance of the processes and the achievement of the ENMSL goals.

Through their human resources process, personnel are identified, selected and incorporated in relation to labor competencies; it diminishes the training time through the DNC, thus developing the competitive characteristics of the ENMSL.

Improvement areas: It is necessary to design a system for participation in innovation and creativity in the improvement of services; stimulating, evaluating, recognizing and rewarding staff for their individual and group contributions in congruence with the principles of ENMSL, this will allow the development of organizational knowledge. 
The implementation of efficient mechanisms to manage the employment relationship will allow the improvement of work systems.

\subsection{Human Development}

Strong areas: The job profiles of the activities carried out according to the organization chart have been identified and defined.

Improvement areas: Mechanisms are needed to create conditions and practices to reinforce continuous learning, this will allow developing organizational knowledge and innovation in its quality system.

The detection of training needs is applied to reduce the gap between the knowledge, skills and attitudes that they have and require in the short and long term, however they are not aligned with organizational strategies.

\subsection{Life Quality}

Strong areas: The academic part promotes stimuli for teachers who develop their skills, the medical services of ISSEG. These are some of the actions for them to have a better life quality, however it is still necessary to create a better life quality system and to assure full family well-being.

Improvement areas: The necessary mechanisms should be identified to define, evaluate and measure the critical satisfaction factors of ENMSL staff. And what actions are carried out to respond to the needs identified with the aim of increasing their life quality and commitment with the institution.

By reactivating the Occupational Safety and Health committee, they can design the mechanisms to promote safety, health, hygiene and ergonomics to all of the ENMSL activities.

\section{Processes}

\subsection{Products Design, Services and Processes}

Improvement areas: The needs and expectations of the customers are known with the satisfaction survey; however, no mechanisms are identified to translate them into characteristics and service specifications to specify a valuable proposal for the organization.

The system responsible for the design, redesign or process improvement that assures a consistent and fault-free performance to respond to the strategic needs of ENMSL is not described.

\subsection{Process Administration}

Improvement areas: Although there is a certified ISO 9000 and GTO-ISO-001 system, it does not show performance indicators, if it had them, this would allow knowing the gaps between the planned and the real in each of the processes and would trigger a continuous improvement of the services in the ENMSL.

\section{Social Responsibility}

\subsection{Ecosystems}

Improvement areas: Through the environmental training program this principle can be fulfilled, however it is necessary to describe systemically the development of each activity: Environmental impact of their processes, optimization of resources, protection and promotion of ecosystem recovery and provide environmental education.

\subsection{Community Development}

Improvement areas: The ISO 26000 and ESR implementation are ambitious projects, however, they must consider that they must do other alternative actions to their duty.

Generate permanent social welfare of their communities of influence, promote and participate with the community in processes of continuous improvement, and share practices in favor of greater competitiveness in the industrial sector, business and the economy in general.

\section{Results}

Improvement areas: It is necessary to place indicators referring to customers, staff and Company with referential comparisons and the causal relationship of the conditions that have been presented in a favorable or unfavorable trend.

\subsection{Value Results created for Shareholders}

Improvement areas: The indicators should show favorable trends, if not, must show the actions that are being taken to correct these deviations.

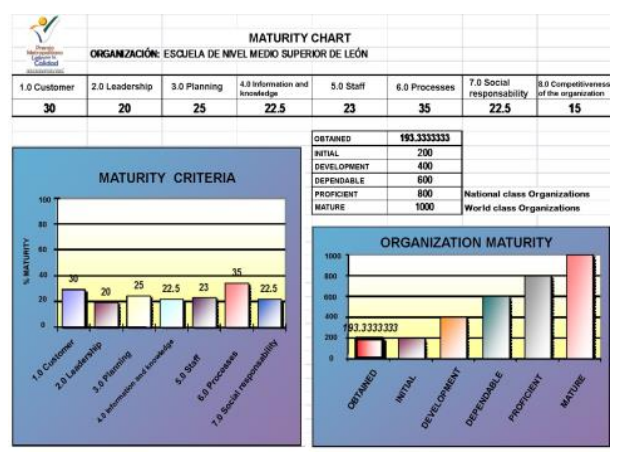

Figure 6. Maturity chart $2^{\text {nd }}$ stage Source: PMLC $2^{\text {nd }}$ stage evaluation report 
As well as complement the indicators to visualize the value created for customers and shareholders.

\section{Conclusions and Recommendations}

1. With reference to ISO 10014: 2006 / NMXCC-10014-IMNC-2008. Quality management Guidelines for obtaining financial and economic benefits.

In absolute terms, the level of maturity of the ENMSL in reference to the application of the eight principles of quality management, presents a maturity above the average, while in relative terms or percentage is scarcely $60 \%$ level of growth.

2. With reference to the PMLC participation guide.

Within the evaluation process of the PMLC, in its first stage of reporting in short, the minimum required score for accreditation was obtained, while in the second stage of systemic reporting, the initial level of maturity for the organization was not even reached. The best-favored criteria for both reports are those focused on processes, clients, leadership and planning, the lowest being the competitiveness of the organization and social responsibility.

3. In reference to both models of quality management.

There are only four identical criteria, which can be aligned in this investigation. It is also clear that they represent two different models, one of quality management system and one of total quality model.

The methods of evaluation and measurement are different, they only share the tools of questionnaires, as a means to measure the level of maturity.

The ISO 10014: 2008 model represents an initial and exhaustive self-assessment, while the PMLC model represents an external evaluation in its first two stages in a descriptive documentary manner in relation to the criteria.

4. Recommendations.

Continue with the measurement of maturity level with ISO 10014: 2006 / NMX-CC-10014-IMNC2008. Quality management - Guidelines for obtaining financial and economic benefits.

Participate in the PMLC again, using the criteria and sub criteria Guide PMLC participation, taking into reference information and the maturity obtained at different stages of this year.

One way of convergence of both models, would be to use the model ISO 9004: 2009 Management for the sustained success of an organization - Focus of quality management, in order to perform a wider self-assessment.

The results obtained in the present investigation will allow the top management of the ENMSL to continue with the continuous improvement in the academic and administrative processes, in order to have better levels of competitiveness in the education sector. Likewise, it is recognized that the results obtained are based on the development of a culture of quality that the institution has permeated in recent years, demonstrated with quality certifications.

Some of the methods and tools that can be used for the principles of quality management that obtained lower level of maturity in this research are open business culture, succession planning, balanced scorecard, comparative studies with best practices , deployment of the quality function, development by competences, among others.

\section{References}

[1] ISO, 2001. Brochure principles of quality management. 3: 1-5.

[2] IMNC, 2005. ISO 9000: 2005 / NMX-CC-9000IMNC-2005. Quality management system Fundamentals and vocabulary. 3: 1-7.

[3] IMNC, 2007. IWA 2: 2007 / NMX-CC-023IMNC-2007. Quality management system Guidelines for the application of ISO 9001: 2008NMX-CC-9001-IMNC-2008 in education. 1: 1-35.

[4] ISO, 2008. ISO / TC 176 N544R2 Process-based approach. 5: 3-6.

[5] IMNC, 2008. ISO 10014: 2006 / NMX-CC10014-IMNC-2008. Quality management Guidelines for obtaining financial and economic benefits.

[6] PMLC. Announcement and guidelines. www.leon.gob.mx/innovacion. 